\title{
Sufficient Conditions for the Existence of Zeno Behavior in a Class of Nonlinear Hybrid Systems via Constant Approximations
}

\author{
Aaron D. Ames, Alessandro Abate and Shankar Sastry
}

\begin{abstract}
The existence of Zeno behavior in hybrid systems is related to a certain type of equilibria, termed Zeno equilibria, that are invariant under the discrete, but not the continuous, dynamics of a hybrid system. In analogy to the standard procedure of linearizing a vector field at an equilibrium point to determine its stability, in this paper we study the local behavior of a hybrid system near a Zeno equilibrium point by considering the value of the vector field on each domain at this point, i.e., we consider constant approximations of nonlinear hybrid systems. By means of these constant approximations, we are able to derive conditions that simultaneously imply both the existence of Zeno behavior and the local exponential stability of a Zeno equilibrium point. Moreover, since these conditions are in terms of the value of the vector field on each domain at a point, they are remarkably easy to verify.
\end{abstract}

\section{INTRODUCTION}

Zeno behavior occurs in a hybrid system if an infinite number of discrete transitions occur in a finite amount of time - that is if, in the limit, the switching times converge. Along with the convergence in time which, by definition, is associated with Zeno behavior, there is also a convergence in space. This convergence is analogous to the classical convergence of trajectories to an equilibrium point, except that the convergence is non-smooth and occurs in finite time. Therefore, when considering Zeno behavior, it is natural to consider the corresponding notion of Zeno equilibria - these are points in the hybrid state space of the hybrid system that are invariant under the discrete dynamics of the hybrid system but not under the continuous dynamics.

Linearizing a nonlinear system about an equilibrium point is a simple and powerful method of determining the stability of that point. Given the analogue between classical equilibria and Zeno equilibria, one naturally arrives at the following question: is there a procedure analogous to linearization that will allow one to determine the stability of Zeno equilibria? Due to the intrinsic relationship between Zeno equilibria and Zeno behavior, the following question also naturally arises: is there a procedure analogous to linearization that will allow one to determine the existence of Zeno behavior?

The goal of this paper is provide a procedure for determining both the stability of Zeno equilibria and the existence of Zeno behavior in a special but illustrative class of nonlinear hybrid systems: first quadrant hybrid systems. As with linearization, this procedure considers the behavior

A. D. Ames is with the Control and Dynamical Systems Department, California Institute of Technology, Pasadena, CA 91125 amesecds. caltech.edu

A. Abate and S. Sastry are with the Department of Electrical Engineering and Computer Sciences, University of California, Berkeley, CA 94720 $\{$ aabate, sastry\}@eecs.berkeley.edu of the vector fields on the domains of the hybrid system near the Zeno equilibrium point. Unlike linearization, this local behavior is encapsulated by the value of these vector fields at the Zeno equilibrium — which must necessarily be nonzerorather than the Jacobian. The main result of this paper is sufficient conditions for the local exponential stability of Zeno equilibria and the existence of Zeno behavior in a class of nonlinear hybrid systems based upon constant approximations, i.e., the value of the vector field on each domain at the Zeno equilibrium point.

Despite the simplicity of the sufficient conditions for Zeno behavior presented in this paper, analyzing Zeno behavior in hybrid systems is notoriously complex and subtle. For this reason, there have been attempts to better understand Zeno behavior by providing necessary conditions (cf. [3], [14]) or sufficient conditions (cf. [2], [9]) for the existence of this behavior. Unfortunately, the necessary conditions obtained to date tend to be extremely conservative and the sufficient conditions tend to be applicable only to very limited classes of hybrid systems, e.g., [9] provides sufficient conditions for Zeno behavior in a class hybrid systems that are, in important ways, different than the systems considered in this paper (this results in conditions that are in a much different spirit than the conditions presented here). Therefore, while the results presented in this paper are applicable only to a limited class of nonlinear hybrid systems, they will hopefully lay the groundwork for better understanding the local nature of Zeno behavior.

\section{FIRST QUADRANT HYBRID SYSTEMS}

We begin this section by considering first quadrant hybrid systems ([1], [2] and [4]); these are nonlinear, twodimensional hybrid systems whose domains and guards display a very specific structure. The motivation for considering hybrid systems of this form is that they are simple enough to be reasoned about yet complex enough to display the type of interesting behavior that is indicative of hybrid systems.

Definition 1: A first quadrant hybrid system, or just FQ hybrid system, is a tuple:

$$
\mathscr{H}=(\Gamma, D, G, R, F),
$$

where

- $\Gamma=(Q, E)$ is a directed cycle graph, with

$$
\begin{aligned}
Q= & \left\{q_{0}, \ldots, q_{k-1}\right\} \\
E= & \left\{e_{0}=\left(q_{0}, q_{1}\right), e_{1}=\left(q_{1}, q_{2}\right),\right. \\
& \left.\ldots, e_{k-1}=\left(q_{k-1}, q_{0}\right)\right\} .
\end{aligned}
$$


- $D=\left\{D_{q}\right\}_{q \in Q}$ is a set of domains, where for all $q \in Q$,

$$
D_{q}=\left(\mathbb{R}_{0}^{+}\right)^{2}=\left\{\left(\begin{array}{l}
x_{1} \\
x_{2}
\end{array}\right) \in \mathbb{R}^{2}: x_{1} \geq 0 \text { and } x_{2} \geq 0\right\} \text {, }
$$

hence the name "first quadrant."

- $G=\left\{G_{e}\right\}_{e \in E}$ is a set of guards, where for all $e \in E$

$$
G_{e}=\left\{\left(\begin{array}{l}
x_{1} \\
x_{2}
\end{array}\right) \in \mathbb{R}^{2}: x_{1}=0 \text { and } x_{2} \geq 0\right\} \text {. }
$$

- $R=\left\{R_{e}\right\}_{e \in E}$ is a set of reset maps, where for all $e \in E$ there exists a function $r_{e}: \mathbb{R}_{0}^{+} \rightarrow \mathbb{R}_{0}^{+}$such that

$$
R_{e}\left(x_{1}, x_{2}\right)=\left(\begin{array}{c}
r_{e}\left(x_{2}\right) \\
x_{1}
\end{array}\right) .
$$

- $F=\left\{f_{q}\right\}_{q \in Q}$ is a set of vector fields, where $f_{q}$ is a Lipschitz vector field on $\mathbb{R}^{2}$.

In this paper, we make the following:

Assumption 1: $r_{e}=$ id for all $e \in E$.

This assumption is not very restrictive since one can make all of the $r_{e}$ the identity map by adding extra domains with corresponding vector fields that mimic the reset maps.

Remark 1: First quadrant hybrid systems are, admittedly, a very restrictive class of hybrid system; again, the purpose of studying these systems is to gain an understanding of Zeno behavior beginning at a simple and fundamental level. That being said, it is possible to consider higher dimensional analogues of first quadrant hybrid systems-defined in the obvious manner-and to extend the conditions presented in this paper to systems of this form. Moreover, it is possible to transform a fairly general class of hybrid systems to these higher dimensional first quadrant hybrid systems by transferring the nonlinearity of the guards to the dynamics by appending a state variable as discussed in [8, Eqn. (1)].

Executions. An (infinite ${ }^{1}$ ) execution of a hybrid system $\mathscr{H}=(\Gamma, D, G, R, F)$ is a tuple $\chi=(I, d, C)$ where

- $I=\left\{I_{i}\right\}_{i \in \mathbb{N}}$ where for each $i \in \mathbb{N}, I_{i}=\left[\tau_{i}, \tau_{i+1}\right]$ with $\tau_{i} \leq \tau_{i+1} \in \mathbb{R}_{0}^{+}$. This is the set of the switching times.

- $d: \mathbb{N} \rightarrow Q$ is a map such that for all $i, i+1 \in \mathbb{N}$, $(d(i), d(i+1)) \in E$. This is the discrete component of the execution.

- $C=\left\{c_{i}\right\}_{i \in \mathbb{N}}$ is a set such that $\dot{c}_{i}(t)=f_{d(i)}\left(c_{i}(t)\right)$ for $t \in I_{i}$. This is a set of continuous trajectories.

We require that for all $i \in \mathbb{N}$,

$$
\begin{gathered}
c_{i}(t) \in D_{d(i)}, \forall t \in I_{i} \\
c_{i}\left(\tau_{i+1}\right) \in G_{(d(i), d(i+1))} \\
c_{i+1}\left(\tau_{i+1}\right)=R_{(d(i), d(i+1))}\left(c_{i}\left(\tau_{i+1}\right)\right) .
\end{gathered}
$$

Note that the continuous initial condition of an execution $\chi$ is, when $I_{0}=\left[\tau_{0}, \tau_{1}\right]$, given by $c_{0}\left(\tau_{0}\right) \in D_{d(0)}$. The discrete initial condition is given by $d(0)$.

The object of study in this paper will be Zeno executions (cf. [10], [14]), which are defined in the following manner:

\footnotetext{
${ }^{1}$ We will consider only infinite executions because of their relevance to the problem under investigation; see [1] and [14] for the definition of finite executions.
}

Definition 2: An execution $\chi$ is Zeno if

$$
\lim _{i \rightarrow \infty} \tau_{i}=\sum_{i=0}^{\infty}\left(\tau_{i+1}-\tau_{i}\right)=\tau_{\infty}
$$

for some finite $\tau_{\infty} \in \mathbb{R}_{0}^{+}$.

A hybrid system $\mathscr{H}$ is Zeno if there exists a Zeno execution $\chi$ with $\tau_{i+1}-\tau_{i} \neq 0$ for some $i \in \mathbb{N}$.

Remark 2: The motivation for defining Zeno hybrid systems as in Def. 2 is that all of the hybrid systems considered in this paper will have an "instantaneously" Zeno execution with initial condition $c_{0}\left(\tau_{0}\right)=0$, i.e., for this execution $\tau_{i+1}=\tau_{i}=0$ for all $i \in \mathbb{N}$. We want to differentiate this type of Zeno behavior from the more interesting types of Zeno behavior, e.g., Zeno executions with $c_{0}\left(\tau_{0}\right) \neq 0$. In this case, there must necessarily exist a $i \in \mathbb{N}$ such that $\tau_{i+1}-\tau_{i} \neq 0$. Other representative types of Zeno behavior, chattering and genuine Zeno, are defined in [1] and [3].

\section{ZENO EQUILIBRIA}

The stability properties of hybrid systems have been studied to a much greater degree than Zeno behavior (cf. [5], [12], [13] and the references therein). Yet Zeno behavior in hybrid systems is fundamentally related to the stability of Zeno equilibria in that the absence of such equilibria implies the absence of Zeno behavior. When studying Zeno behavior, therefore, it is natural to also study Zeno equilibria and the stability properties thereof; see [1] and [4] for a definition of Zeno equilibria for general hybrid systems.

Definition 3: The point $x=0$ is a Zeno equilibrium point of $\mathscr{H}$ in Def. 1 if $f_{q}(0) \neq 0$ for all $q \in Q$.

We now introduce a type of stability that Zeno equilibrium points can display, termed local exponential stability, that directly relates to Zeno behavior. We will provide evidence of this fact in Theorem 3, where the conditions that imply Zeno behavior are the same conditions that imply the local exponential stability of Zeno equilibria.

Definition 4: The Zeno equilibrium point $x=0$ of $\mathscr{H}$ is locally exponentially stable if for any set $\left\{\varepsilon_{q}\right\}_{q \in Q}$ with $\varepsilon_{q}>0$, there exists a $\delta>0$ such that for every execution $\chi=$ $(I, d, C)$ satisfying $\left\|c_{0}\left(\tau_{0}\right)\right\|<\delta$ the following conditions hold:

S1: $\quad\left\|c_{i}(t)\right\|<\varepsilon_{d(i)}$ for all $t \in I_{i}$ and $i \in \mathbb{N}$.

S2: $\quad\left\|c_{i}\left(\tau_{i}\right)\right\| \leq M\left\|c_{1}\left(\tau_{1}\right)\right\| \gamma^{i-1}$. for all $i \in \mathbb{N} \backslash\{0\}$, where $M \geq 1$ and $0<\gamma<1$.

Note that $\mathbf{S 1}$ guarantees that the continuous portion of the execution must remain bounded for all time, while S2 guarantees that the discrete portion of the execution must converge exponentially to the origin. In addition, we utilize $c_{1}\left(\tau_{1}\right)$ in condition $\mathbf{S 2}$ because this point is necessarily on the $x_{1}$ axis, while $c_{0}\left(\tau_{0}\right)$ is not necessarily on this axis. That is, $\mathbf{S 2}$ enforces exponential stability on a set of points on the $x_{1}$-axis.

\section{EXISTENCE OF EVENTS}

In this section, we give conditions on the existence of events on a single domain of $\mathscr{H}$ which will be used in the next section to prove the existence of infinite executions. 
Existence of events. Consider the vector field $\dot{x}=f_{q}(x)$ for some $q \in Q$. We say that there exists an event for $f_{q}$ and $x_{0} \in D_{q}$ if for the solution $c(t)$ of $\dot{x}=f_{q}(x)$ on $\left[t_{0}, t_{1}\right]$ with $c\left(t_{0}\right)=x_{0}$ the following conditions hold:

E1: There exists a time $\tau \leq t_{1}$ such that $c(\tau) \in G_{e}$, with $e \in E$ the unique edge such that the source of $e$ is $q$.

E2: For all $t \in\left[t_{0}, \tau\right], c(t) \in D_{q}$.

The following theorem relates the value of the vector field at the origin, $f_{q}(0)$, to the existence of events. First, note that we denote a ball around the origin of radius $r$ by $B(r, 0)=$ $\left\{x \in \mathbb{R}^{2}:\|x\| \leq r\right\}$, and we denote the $i^{\text {th }}$ component of $f_{q}(x)$ by $\left(f_{q}(x)\right)_{i}$, for $i=1,2$.

Theorem 1: There exists an event for $\dot{x}=f_{q}(x)$ and for all $x_{0} \in D_{q} \cap B\left(r_{q}, 0\right)$, with $r_{q}>0$ sufficiently small, if

$$
\begin{gathered}
\left(f_{q}(0)\right)_{1}<0<\left(f_{q}(0)\right)_{2}, \\
-\frac{\left(f_{q}(0)\right)_{2}}{\left(f_{q}(0)\right)_{1}}<1 .
\end{gathered}
$$

To prove this theorem, we will need to utilize the classic bound on the difference between solutions of comparable ordinary differential equations, as can be found in [6, Theorem 2.1] and [11, Theorem 3.4].

The solution comparison theorem. Consider the vector field $\dot{x}=f_{q}(x)$. A smooth $\varepsilon$-approximate solution to this vector field on a time interval $I=\left[t_{0}, t_{1}\right]$ is a smooth function $\varphi: I \rightarrow \mathbb{R}^{2}$ such that:

$$
\left\|\dot{\varphi}(t)-f_{q}(\varphi(t))\right\| \leq \varepsilon
$$

for all $t \in I$. If $c(t)$ is the solution to $f_{q}$ and $c\left(t_{0}\right)=\varphi\left(t_{0}\right)$, then for all $t \in I$ :

$$
\|\varphi(t)-c(t)\| \leq \frac{\varepsilon}{L_{q}}\left(e^{L_{q} t}-1\right),
$$

where $L_{q}$ is the Lipschitz constant of $f_{q}$.

Proof: [of Theorem 1] We will prove the theorem in the case when $x_{0}=(\rho, 0)^{T}$, for $0 \leq \rho<\tilde{\rho}$ with $\tilde{\rho}>0$, i.e., for $x_{0}$ on a subset the $x_{1}$-axis. It is a simple matter to extend the arguments used to prove the case when $x_{0}=(\rho, 0)^{T}$ to the case when $x_{0} \neq(\rho, 0)^{T}$ by utilizing the continuity of $f_{q}$; space constraints prevent the inclusion of these details.

We exploit the above comparison theorem by taking $\varphi(t)=f_{q}(0) t+(\rho, 0)^{T}$, i.e, the solution to $\dot{x}=f_{q}(0)$. By the Lipschitz continuity of $f_{q}$, we have that

$$
\left\|\dot{\varphi}(t)-f_{q}(\varphi(t))\right\| \leq L_{q}\left\|f_{q}(0) t+(\rho, 0)^{T}\right\| .
$$

The first step is to find the values of $t$ such that

$$
\left\|f_{q}(0) t+(\rho, 0)^{T}\right\| \leq \rho,
$$

which will ensure that

$$
\left\|\dot{\varphi}(t)-f_{q}(\varphi(t))\right\| \leq L_{q} \rho .
$$

To check when the inequality in (2) holds, we find the points where the equality holds, which are

$$
t_{0}=0, \quad t_{1}(\rho)=-\frac{2\left(f_{q}(0)\right)_{1} \rho}{\left(f_{q}(0)\right)_{1}^{2}+\left(f_{q}(0)\right)_{2}^{2}},
$$

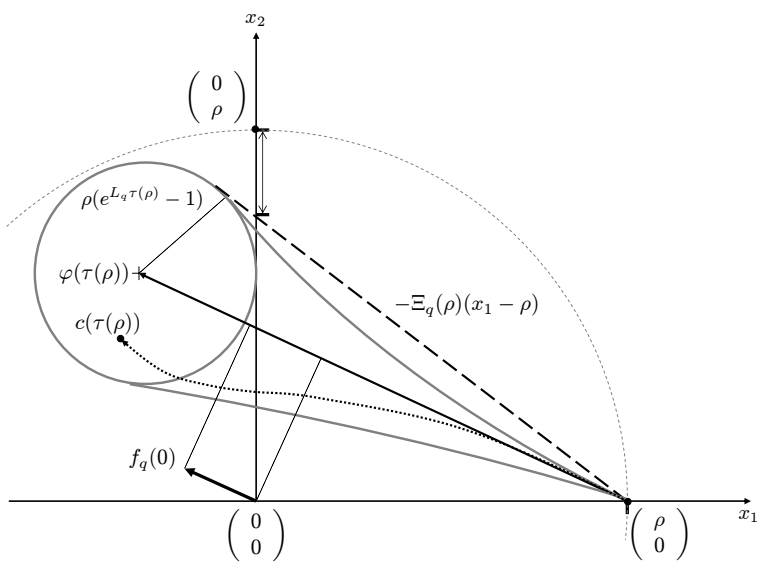

Fig. 1. The error cone around $\varphi(t)$.

and it follows that $t_{1}(\rho)$ is positive for $\rho>0$ by the assumption that $\left(f_{q}(0)\right)_{1}<0$. Therefore, for all $t \in\left[0, t_{1}(\rho)\right]$, (2) holds and thus (3) holds. It follows by the solution comparison theorem that, for all $t \in\left[0, t_{1}(\rho)\right]$,

$$
\|\varphi(t)-c(t)\| \leq \rho\left(e^{L_{q} t}-1\right) .
$$

The main observation of this proof is that if there exists a $\tau(\rho) \in\left[0, t_{1}(\rho)\right]$, which is dependent on $\rho$, such that

$$
-\left(\left(f_{q}(0)\right)_{1} \tau(\rho)+\rho\right)=\rho\left(e^{L_{q} \tau(\rho)}-1\right),
$$

then there must exist a $\hat{\tau}(\rho) \leq \tau(\rho)$ (also dependent on $\rho$ ) such that $c(t)$ has an event at $\hat{\tau}(\rho)$ with $c\left(t_{0}\right)=\varphi\left(t_{0}\right)=$ $(\rho, 0)^{T}$, i.e., $\mathbf{E 1}$ and $\mathbf{E 2}$ are satisfied (see Fig. 1 for a graphical interpretation).

Condition $\mathbf{E} 1$ follows from the fact that at time $\tau(\rho)$, the error ball of radius $\rho\left(e^{L_{q} \tau(\rho)}-1\right)$, centered at $\varphi(\tau(\rho))$, has completely crossed the $x_{2}$-axis. Since $c(\tau(\rho))$ is contained in this ball by (5), it must as well have crossed the $x_{2}$-axis, and hence there must have been an event.

Condition $\mathbf{E} 2$ follows from the fact that, by the continuity of $f_{q},\left(f_{q}(\rho, 0)\right)_{2}>0$ for all $\rho>0$ sufficiently small. This, in turn, implies that there exists a $\rho_{c}>0$ such that $c(t) \in D_{q}$ for all $t \in[0, \hat{\tau}(\rho)]$ for all $0 \leq \rho<\rho_{c}$.

To complete the proof, we must establish the existence of a $\tau(\rho) \in\left[0, t_{1}(\rho)\right]$ satisfying (6). Solving (6) for $\tau(\rho)$ yields:

$$
\tau(\rho)=-\frac{1}{L_{q}} \mathcal{W}\left(\frac{L_{q} \rho}{\left(f_{q}(0)\right)_{1}}\right),
$$

where $\mathcal{W}$ is the Lambert $W$ function (see [7] and the references therein). Note that $\tau(\rho)$ is well-defined for all $0 \leq \rho \leq-\frac{\left(f_{q}(0)\right)_{1}}{L_{q} e}$. It is easy to verify that that $\tau(\rho) \geq 0$ for these values since $\mathcal{W}(z) \leq 0$ for $z \in[-1 / e, 0]$. Also note that $\mathcal{W}$ is a concave function of its arguments, and hence $\tau(\rho)$ is a convex function of $\rho$.

Now we need to verify for certain values of $\rho$ that $\tau(\rho) \in$ $\left[0, t_{1}(\rho)\right]$. To find these values of $\rho$, we begin by solving for $\rho$ in the following equation: $t_{1}(\rho)=\tau(\rho)$, which has two solutions: $\rho_{0}=0$ and

$$
\rho_{1}=\frac{\left(\left(f_{q}(0)\right)_{1}^{2}+\left(f_{q}(0)\right)_{2}^{2}\right) \log \left(\frac{\left(f_{q}(0)\right)_{1}^{2}+\left(f_{q}(0)\right)_{2}^{2}}{2\left(f_{q}(0)\right)_{1}^{2}}\right)}{2\left(f_{q}(0)\right)_{1} L_{q}} .
$$


We claim that $\tau(\rho) \in\left[0, t_{1}(\rho)\right]$ for $0 \leq \rho \leq \rho_{1}$. But this follows directly from the fact that $t_{1}(\rho)$ is a linear function of $\rho, \tau(\rho)$ is a convex function of $\rho$ and $\rho_{1}$ satisfies $\tau\left(\rho_{1}\right)=$ $t_{1}\left(\rho_{1}\right)$. This is equivalent to saying that the curve $\tau(\rho)$ lies below the line $t_{1}(\rho)$ for all values of its argument in the interval $0 \leq \rho \leq \rho_{1}$.

As a result of the above arguments, we have established that for all $0 \leq \rho<\tilde{\rho}=\min \left\{\rho_{c}, \rho_{1}\right\}$, there exists an event for all $x_{0}=(\rho, 0)^{T}$.

\section{EXISTENCE OF EXECUTIONS}

In this section, we establish the existence of infinite executions and demonstrate that these executions display several important properties.

The "event approximation" function. Consider the error cone that propagates around the solution of the constant vector field $\dot{x}=f_{q}(0)$, starting at $(\rho, 0)^{T}$ with $\rho>0$ sufficiently small, according to the error bound in (5) (see Fig. 1 for a pictorial representation). Under the assumptions of Theorem 1, we know that there exists an event for the nonlinear vector field $\dot{x}=f_{q}(x)$, i.e., a time $\tau^{*}$ such that $\mathbf{E} 1$ and $\mathbf{E 2}$ are satisfied. The goal is to find an upper bound for $\left(c\left(\tau^{*}\right)\right)_{2}$ through the error cone obtained from (5).

Let $\varphi(t)=f_{q}(0) t+(\rho, 0)^{T}$ be the solution to $\dot{x}=f_{q}(0)$ with initial condition $(\rho, 0)^{T}$. Define a straight line in the plane passing through the point $(\rho, 0)^{T}$ and lying tangent to the circle centered at $\varphi(\tau(\rho))$, where $\tau(\rho)$ is given as in (7), with radius $r(\rho)=\rho\left(e^{L_{q} \tau(\rho)}-1\right)$; a visual interpretation can, again, be found in Fig. 1. This defines a line given by:

$$
x_{2}=-\Xi_{q}(\rho)\left(x_{1}-\rho\right),
$$

where its slope $\Xi_{q}(\rho)$ is given, as a function of $\rho$, by

$$
\begin{aligned}
& \Xi_{q}(\rho)= \\
& \frac{\left(f_{q}(0)\right)_{1}-\left(f_{q}(0)\right)_{2} \sqrt{1+\frac{\left(f_{q}(0)\right)_{2}^{2}}{\left(f_{q}(0)\right)_{1}^{2}}}-\left(f_{q}(0)\right)_{1} e^{\mathcal{W}\left(\frac{L_{q} \rho}{\left(f_{q}(0)\right)_{1}}\right)}}{\left(f_{q}(0)\right)_{2}+\left(f_{q}(0)\right)_{1} \sqrt{1+\frac{\left(f_{q}(0)\right)_{2}^{2}}{\left(f_{q}(0)\right)_{1}^{2}}}-\left(f_{q}(0)\right)_{2} e^{\mathcal{W}\left(\frac{L_{q} \rho}{\left(f_{q}(0)\right)_{1}}\right)}},
\end{aligned}
$$

where, again, $\mathcal{W}$ is the Lambert $\mathrm{W}$ function.

We term $\Xi_{q}(\rho)$ the event approximation function. It has the following important properties:

Lemma 1: For $\Xi_{q}(\rho)$ as defined in (8), if

$$
\begin{gathered}
\left(f_{q}(0)\right)_{1}<0<\left(f_{q}(0)\right)_{2}, \\
-\frac{\left(f_{q}(0)\right)_{2}}{\left(f_{q}(0)\right)_{1}}<1,
\end{gathered}
$$

then the following properties hold:

- $\Xi_{q}(\rho)$ is continuous and strictly increasing as a function of $\rho$ for all $0 \leq \rho \leq-\frac{\left(f_{q}(0)\right)_{1}}{L_{q} e}$.

- There exists a $\hat{\rho}_{q}>0$ such that

$$
-\frac{\left(f_{q}(0)\right)_{2}}{\left(f_{q}(0)\right)_{1}} \leq \Xi_{q}(\rho)<1
$$

for all $\rho \in\left[0, \hat{\rho}_{q}\right)$.

Proof: The continuity of $\Xi_{q}(\rho)$ is easy to verify on the values for which it is defined. Note that the domain of definition of this function is dictated by the domain of definition of the Lambert $\mathrm{W}$ function.

To verify that $\Xi_{q}(\rho)$ is strictly increasing, we consider its derivative and verify that it is always strictly positive. By direct calculation, $\frac{d \Xi_{q}}{d \rho}$ is given as in (10). Now, the numerator in (10) is clearly negative, so we need to verify that the denominator is also negative. Since, by assumption, $\left(f_{q}(0)\right)_{1}<0$, this amounts to checking that

$$
\left(1+\mathcal{W}\left(\frac{L_{q} \rho}{\left(f_{q}(0)\right)_{1}}\right)\right)>0, \quad \rho \in\left[0,-\frac{\left(f_{q}(0)\right)_{1}}{L_{q} e}\right] .
$$

But this is equivalent to checking that $\mathcal{W}(z) \geq-1, z \in$ $[-1 / e, 0]$, which follows from the properties of the Lambert $\mathrm{W}$ function.

To prove the final property of $\Xi_{q}$, we note that

$$
\Xi_{q}(0)=-\frac{\left(f_{q}(0)\right)_{2}}{\left(f_{q}(0)\right)_{1}}<1
$$

Therefore, by the continuity of $\Xi_{q}$, it follows that there exists a sufficiently small $\hat{\rho}_{q}>0$ such that (9) holds for all $\rho \in$ $\left[0, \hat{\rho}_{q}\right)$.

We now have the necessary framework in which to prove the main result of this section.

Theorem 2: Let $\mathscr{H}=(\Gamma, D, G, R, F)$ be a FQ hybrid system satisfying

$$
\begin{gathered}
\left(f_{q}(0)\right)_{1}<0<\left(f_{q}(0)\right)_{2}, \\
-\frac{\left(f_{q}(0)\right)_{2}}{\left(f_{q}(0)\right)_{1}}<1 .
\end{gathered}
$$

for all $q \in Q$. Then for any set $\left\{\varepsilon_{q}\right\}_{q \in Q}$ with $\varepsilon_{q}>0$, there exists a $\delta>0$ such that for all $x_{0} \in\left(\mathbb{R}_{0}^{+}\right)^{2}$ satisfying $\left\|x_{0}\right\|<\delta$, there exists a unique execution $\chi=(I, d, C)$ with $c_{0}\left(\tau_{0}\right)=x_{0}$ satisfying the following conditions:

(T1) For all $t \in I_{i}$ and $i \in \mathbb{N}$,

$$
\left\|c_{i}(t)\right\|<\varepsilon_{d(i)} .
$$

(T2) For all ${ }^{2} i \in \mathbb{N} \backslash\{0\}$,

$$
\left\|c_{i+1}\left(\tau_{i+1}\right)\right\| \leq\left(\prod_{n=1}^{i} \Xi_{d(n)}\left(\left\|c_{1}\left(\tau_{1}\right)\right\|\right)\right)\left\|c_{1}\left(\tau_{1}\right)\right\| .
$$

Proof: Suppose we are given a set $\left\{\varepsilon_{q}\right\}_{q \in Q}$ with $\varepsilon_{q}>$ 0 . The first step is to construct the $\delta>0$ to be utilized in the theorem.

Recall that by Theorem 1, for all $q \in Q$, there exists an $r_{q}>0$ such that there exists an event for $\dot{x}=f_{q}(x)$ for all $x_{0} \in D_{q} \cap B\left(r_{q}, 0\right)$. For the sake of definiteness, let $c\left(t ; x_{0}\right)$ denote the solution of $\dot{x}=f_{q}(x)$ with $c(0)=x_{0}$ and let $\tau_{q}\left(x_{0}\right)$ denote the time at which the event occurs (as given in E1 and E2); here we make the dependence of $\tau$ on $q$ and $x_{0}$ explicit. Let $\beta_{q}$ be the constant such that

$$
\sup _{x_{0} \in D_{q} \cap B\left(\beta_{q}, 0\right)} \max _{t \in\left[0, \tau_{q}\left(x_{0}\right)\right]}\left\|c\left(t ; x_{0}\right)\right\| \leq \min _{q \in Q}\left\{\hat{\rho}_{q}, r_{q}, \varepsilon_{q}\right\},
$$

where $r_{q}$ is defined as in Theorem 1 and $\hat{\rho}_{q}, q \in Q$, are the constants given in Lemma 1. Note that $\beta_{q}$ is welldefined since we are considering smooth functions over finite

\footnotetext{
${ }^{2}$ Note that $\left\|c_{i}\left(\tau_{i}\right)\right\|=\left(c_{i}\left(\tau_{i}\right)\right)_{1}$.
} 


$$
\frac{d \Xi_{q}}{d \rho}=\frac{-L_{q}\left(\left(f_{q}(0)\right)_{1}^{2}+\left(f_{q}(0)\right)_{2}^{2}\right) \sqrt{1+\frac{\left(f_{q}(0)\right)_{2}^{2}}{\left(f_{q}(0)\right)_{1}^{2}}}}{\left(f_{q}(0)\right)_{1}\left(\left(f_{q}(0)\right)_{2}+\left(f_{q}(0)\right)_{1} \sqrt{1+\frac{\left(f_{q}(0)\right)_{2}^{2}}{\left(f_{q}(0)\right)_{1}^{2}}}-\left(f_{q}(0)\right)_{2} e^{\left.\mathcal{W}\left(\frac{L_{q} \rho}{\left(f_{q}(0)\right)_{1}}\right)\right)^{2}}\left(1+\mathcal{W}\left(\frac{L_{q} \rho}{\left(f_{q}(0)\right)_{1}}\right)\right)\right.}
$$

intervals and clearly $0<\beta_{q} \leq \min _{q \in Q}\left\{\hat{\rho}_{q}, r_{q}, \varepsilon_{q}\right\}$. We thus define

$$
\delta=\min _{q \in Q}\left\{\beta_{q}\right\}
$$

wherein it follows that $\delta \leq \min _{q \in Q}\left\{\hat{\rho}_{q}, r_{q}, \varepsilon_{q}\right\}$.

Without loss of generality we will consider executions with the discrete initial condition $d(0)=q_{0}$. That is, we will show the existence of an execution $\chi=(I, d, C)$ with $d(i):=q_{i(\bmod k)}$, satisfying conditions $\mathbf{T 1}$ and $\mathbf{T 2}$, through induction on $i \in \mathbb{N}$. Note that for the rest of the proof, we will let $c_{i}(t)$ denote the solution to $\dot{x}=f_{d(i)}(x)$.

Let $x_{0}$ satisfy $\left\|x_{0}\right\|<\delta$. Picking $c_{0}\left(\tau_{0}\right)=x_{0}$, since $\delta \leq$ $r_{q_{0}}$ it follows that there exists time $\tau_{1}$ such that $c_{0}\left(\tau_{1}\right) \in$ $G_{\left(q_{0}, q_{1}\right)}$, i.e., at $\tau_{1}, c_{0}\left(\tau_{1}\right)=\left(0,\left(c_{0}\left(\tau_{1}\right)\right)_{2}\right)^{T}$. Moreover, it follows by the definition of $\beta_{q_{0}}$ that $\|c(t)\|<\varepsilon_{q_{0}}$ for all $t \in\left[\tau_{0}, \tau_{1}\right]$. Therefore, condition $\mathbf{T 1}$ is satisfied for $i=0$. (Note that condition $\mathbf{T} 2$ does not depend on $c_{0}\left(\tau_{0}\right)$, so we do not need to verify it in the case when $i=0$.)

Setting $c_{1}\left(\tau_{1}\right)=\left(\left(c_{0}\left(\tau_{1}\right)\right)_{2}, 0\right)^{T}$, it follows that:

$$
\left(c_{1}\left(\tau_{1}\right)\right)_{1}<\hat{\rho}_{q_{1}}, \quad\left\|c_{1}\left(\tau_{1}\right)\right\|=\left\|c_{0}\left(\tau_{1}\right)\right\|<r_{q_{1}} .
$$

Therefore, by Theorem 1 there exists a time $\tau_{2}$ such that $c_{1}\left(\tau_{2}\right) \in G_{\left(q_{1}, q_{2}\right)}$, i.e., at $\tau_{2}, c_{1}\left(\tau_{2}\right)=\left(0,\left(c_{1}\left(\tau_{2}\right)\right)_{2}\right)^{T}$. Moreover, by construction of the function $\Xi_{q_{1}}$, it follows that $\left(c_{1}\left(\tau_{2}\right)\right)_{2}$ is bounded above by the point at which the line

$$
x_{2}=-\Xi_{q_{1}}\left(\left(c_{1}\left(\tau_{1}\right)\right)_{1}\right)\left(x_{1}-\left(c_{1}\left(\tau_{1}\right)\right)_{1}\right)
$$

intersects the $x_{2}$-axis. That is,

$$
\left(c_{1}\left(\tau_{2}\right)\right)_{2} \leq \Xi_{q_{1}}\left(\left(c_{1}\left(\tau_{1}\right)\right)_{1}\right)\left(c_{1}\left(\tau_{1}\right)\right)_{1}<\left(c_{1}\left(\tau_{1}\right)\right)_{1}
$$

by (9) in Lemma 1. Setting $c_{2}\left(\tau_{2}\right)=\left(\left(c_{1}\left(\tau_{2}\right)\right)_{2}, 0\right)^{T}$, it follows that

$$
\left\|c_{2}\left(\tau_{2}\right)\right\| \leq \Xi_{q_{1}}\left(\left\|c_{1}\left(\tau_{1}\right)\right\|\right)\left\|c_{1}\left(\tau_{1}\right)\right\| .
$$

Therefore, we have established condition $\mathbf{T} 2$ in the case when $i=1$.

In addition, by the construction of $\Xi_{q_{1}}$ and the arguments utilized in Theorem 1, it follows that for all $t \in\left[\tau_{1}, \tau_{2}\right]$

$$
\begin{aligned}
& c_{1}(t) \in\left\{x \in\left(\mathbb{R}_{0}^{+}\right)^{2}:\right. \\
&\left.x_{2} \leq-\Xi_{q_{1}}\left(\left(c_{1}\left(\tau_{1}\right)\right)_{1}\right)\left(x_{1}-\left(c_{1}\left(\tau_{1}\right)\right)_{1}\right)\right\} .
\end{aligned}
$$

Therefore, for all $t \in\left[\tau_{1}, \tau_{2}\right]$,

$$
\left\|c_{1}(t)\right\| \leq\left\|c_{1}\left(\tau_{1}\right)\right\|=\left\|c_{0}\left(\tau_{1}\right)\right\|<\varepsilon_{q_{1}},
$$

by the definition of $\beta_{q_{0}}$. Thus, T1 is satisfied for $i=1$.

Now, by way of induction, suppose that:

- There exists an interval $I_{i-1}=\left[\tau_{i-1}, \tau_{i}\right]$ such that (i), (ii) and (iii) in (1) are satisfied.
- For all $t \in I_{i},\left\|c_{i-1}(t)\right\| \leq \varepsilon_{d(i-1)}$.

- $\left\|c_{i}\left(\tau_{i}\right)\right\| \leq\left(\prod_{n=1}^{i-1} \Xi_{d(n)}\left(\left\|c_{1}\left(\tau_{1}\right)\right\|\right)\right)\left\|c_{1}\left(\tau_{1}\right)\right\|$.

Take $c_{i}\left(\tau_{i}\right)=\left(\left(c_{i-1}\left(\tau_{i}\right)\right)_{2}, 0\right)^{T}$. Since, by (9),

$$
\begin{aligned}
\left\|c_{i}\left(\tau_{i}\right)\right\| & \leq\left(\prod_{n=1}^{i-1} \Xi_{d(n)}\left(\left(c_{1}\left(\tau_{1}\right)\right)_{1}\right)\right)\left(c_{1}\left(\tau_{1}\right)\right)_{1} \\
& <\left\|c_{1}\left(\tau_{1}\right)\right\|=\left\|c_{0}\left(\tau_{1}\right)\right\| \\
& <r_{d(i)},
\end{aligned}
$$

it follows from Theorem 1 that there exists a time $\tau_{i+1}$ such that $c_{i}\left(\tau_{i+1}\right) \in G_{(d(i), d(i+1))}$, or $c_{i}\left(\tau_{i+1}\right)=$ $\left(0,\left(c_{i}\left(\tau_{i+1}\right)\right)_{2}\right)^{T}$. Moreover, $\left(c_{i}\left(\tau_{i+1}\right)\right)_{2}$ is bounded above by the point at which the line

$$
x_{2}=-\Xi_{d(i)}\left(\left(c_{i}\left(\tau_{i}\right)\right)_{1}\right)\left(x_{1}-\left(c_{i}\left(\tau_{i}\right)\right)_{1}\right)
$$

intersects the $x_{2}$-axis. In particular, by the properties of $\Xi_{d(i)}$ stated in Lemma 1, it follows that $\left\|c_{i}\left(\tau_{i}\right)\right\|<\left\|c_{1}\left(\tau_{1}\right)\right\|$ so

$$
\begin{aligned}
& \left\|c_{i+1}\left(\tau_{i+1}\right)\right\| \\
& \quad \leq \Xi_{d(i)}\left(\left\|c_{i}\left(\tau_{i}\right)\right\|\right)\left\|c_{i}\left(\tau_{i}\right)\right\| \\
& \leq \Xi_{d(i)}\left(\left\|c_{1}\left(\tau_{1}\right)\right\|\right)\left(\prod_{n=1}^{i-1} \Xi_{d(n)}\left(\left\|c_{1}\left(\tau_{1}\right)\right\|\right)\right)\left\|c_{1}\left(\tau_{1}\right)\right\| \\
& \quad=\left(\prod_{n=1}^{i} \Xi_{d(n)}\left(\left\|c_{1}\left(\tau_{1}\right)\right\|\right)\right)\left\|c_{1}\left(\tau_{1}\right)\right\| .
\end{aligned}
$$

Therefore, we have established condition $\mathbf{T} 2$ by induction.

Finally, again by the construction of $\Xi_{d(i)}$ and the arguments utilized in Theorem 1, it follows that

$$
\begin{aligned}
c_{i}(t) \in\left\{x \in\left(\mathbb{R}_{0}^{+}\right)^{2}:\right. & \\
& \left.x_{2} \leq-\Xi_{d(i)}\left(\left(c_{i}\left(\tau_{i}\right)\right)_{1}\right)\left(x_{1}-\left(c_{i}\left(\tau_{i}\right)\right)_{1}\right)\right\} .
\end{aligned}
$$

for all $t \in I_{i}=\left[\tau_{i}, \tau_{i+1}\right]$. Therefore, for all $t \in I_{i}$,

$$
\left\|c_{i}(t)\right\| \leq\left\|c_{i}\left(\tau_{i}\right)\right\|<\left\|c_{1}\left(\tau_{1}\right)\right\|=\left\|c_{0}\left(\tau_{0}\right)\right\|<\varepsilon_{d(i)}
$$

by the properties of $\beta_{q_{0}}$. Thus, we have established the existence of an infinite execution with properties $\mathbf{T 1}$ and $\mathbf{T 2}$.

\section{Sufficient Conditions For the Existence of

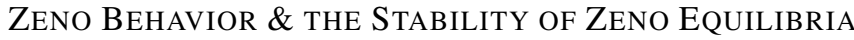

In this section, we present sufficient conditions for the existence of Zeno behavior in a hybrid system based upon the value of the vector field in each domain at the Zeno equilibrium point. In addition, we demonstrate that the same conditions that imply Zeno behavior imply the local exponential stability of the Zeno equilibria $z=0$. This is the main result of this paper. 
Theorem 3: Let $\mathscr{H}=(\Gamma, D, G, R, F)$ be a $\mathbf{F Q}$ hybrid system. If for all $q \in Q$,

$$
\begin{gathered}
\left(f_{q}(0)\right)_{1}<0<\left(f_{q}(0)\right)_{2}, \\
-\frac{\left(f_{q}(0)\right)_{2}}{\left(f_{q}(0)\right)_{1}}<1,
\end{gathered}
$$

then

- $\mathscr{H}$ is Zeno.

- The Zeno equilibria $z=0$ is locally exponentially stable.

Proof: For the sake of simplicity (and due to space constraints), we will prove the theorem in the case when $Q=\{q\}$ and $E=\{e=(q, q)\}$, i.e., in the case when there is a single domain and edge. The same general reasoning can be applied to arbitrary directed cycles.

We begin by proving that $\mathscr{H}$ is Zeno. Let $\tilde{\rho}_{q}$ be the constant given in Theorem 1, let $\hat{\rho}_{q}$ be the constant given in Lemma 1 and let $\varepsilon_{q}=\min \left\{\hat{\rho}_{q}, \tilde{\rho}_{q}\right\}$ be the constant considered in the statement of Theorem 2 . Then there exists a $\rho>0$ such that there exists an execution $\chi=(I, d, C)$ with $c_{0}\left(\tau_{0}\right)=(\rho, 0)^{T}$ (here $\left.d(i) \equiv q\right)$. Moreover, by Theorem 2 , $\left\|c_{i}(t)\right\|<\varepsilon_{q}=\tilde{\rho}_{q}$ implies that $\left\|c_{i}\left(\tau_{i}\right)\right\|<\tilde{\rho}_{q}$ and

$$
\left\|c_{i+1}\left(\tau_{i+1}\right)\right\| \leq\left(\prod_{n=0}^{i} \Xi_{q}(\rho)\right) \rho=\Xi_{q}(\rho)^{i+1} \rho
$$

where here, $\left\|c_{0}\left(\tau_{0}\right)\right\|=\rho$ and we utilize $\left\|c_{0}\left(\tau_{0}\right)\right\|$ rather than $\left\|c_{1}\left(\tau_{1}\right)\right\|$ because $c_{0}\left(\tau_{0}\right)$ is on the $x_{1}$-axis.

Now, since $\left\|c_{i}\left(\tau_{i}\right)\right\|<\tilde{\rho}_{q}$ and, by the same arguments utilized in Theorem 1, there exists a $\Delta \hat{\tau}_{i}$ such that

$$
-\left(\left(f_{q}(0)\right)_{1} \Delta \hat{\tau}_{i}+\left\|c_{i}\left(\tau_{i}\right)\right\|\right)=\left\|c_{i}\left(\tau_{i}\right)\right\|\left(e^{L_{q} \Delta \hat{\tau}_{i}}-1\right),
$$

therefore,

$$
\tau_{i+1}-\tau_{i} \leq \Delta \hat{\tau}_{i}=-\frac{1}{L_{q}} \mathcal{W}\left(\frac{L_{q}\left\|c_{i}\left(\tau_{i}\right)\right\|}{\left(f_{q}(0)\right)_{1}}\right) .
$$

The Lambert $\mathrm{W}$ function satisfies the important inequality (cf. [7]) $W(z) \leq z$ for all $z \in[-1 / e, \infty)$. Therefore,

$$
\begin{aligned}
\sum_{i=0}^{\infty} \tau_{i+1}-\tau_{i} & \leq \sum_{i=0}^{\infty} \Delta \hat{\tau}_{i} \\
& =\sum_{i=0}^{\infty}-\frac{1}{L_{q}} \mathcal{W}\left(\frac{L_{q}\left\|c_{i}\left(\tau_{i}\right)\right\|}{\left(f_{q}(0)\right)_{1}}\right) \\
& \leq \sum_{i=0}^{\infty}-\frac{\left\|c_{i}\left(\tau_{i}\right)\right\|}{\left(f_{q}(0)\right)_{1}} \\
& \leq-\frac{1}{\left(f_{q}(0)\right)_{1}} \sum_{i=0}^{\infty} \Xi_{q}(\rho)^{i} \rho \\
& =-\frac{\rho}{\left(f_{q}(0)\right)_{1}\left(1-\Xi_{q}(\rho)\right)}
\end{aligned}
$$

since $\Xi_{q}(\rho)<1$ by Lemma 1 because $\left\|c_{0}\left(\tau_{0}\right)\right\|=\rho<\varepsilon_{q} \leq$ $\hat{\rho}_{q}$. Therefore, the execution $\chi$ is Zeno, and so $\mathscr{H}$ is Zeno.

Now, we will prove that $z=0$ is a locally exponentially stable Zeno equilibrium. Let $\varepsilon_{q}>0$ be given. By Theorem 2, there exists a $\delta>0$ such that for all $x_{0}$ satisfying $\left\|x_{0}\right\|<\delta$ there exists an execution $\chi=(I, d, C)$ such that $c_{0}\left(\tau_{0}\right)=$ $x_{0}$ satisfying the properties given in Theorem 2 . In fact, by construction, it follows that every execution $\chi=(I, d, C)$ satisfying $\left\|c_{0}\left(\tau_{0}\right)\right\|<\delta$ must satisfy the properties given in Theorem 2. We have thus already established $\mathbf{S 1}$ and need only show that, for every such execution, $\mathbf{S 2}$ is satisfied.

Consider an execution $\chi=(I, d, C)$ satisfying $\left\|c_{0}\left(\tau_{0}\right)\right\|<$ $\delta$. By the properties of $\Xi_{q}$ given in Lemma 1, we have that, for all $i \in \mathbb{N} \backslash\{0\}$,

$$
\left\|c_{i+1}\left(\tau_{i+1}\right)\right\| \leq \Xi_{q}\left(\left\|c_{i}\left(\tau_{i}\right)\right\|\right)\left\|c_{i}\left(\tau_{i}\right)\right\|
$$

as was utilized in the proof of Theorem 2. If we, therefore, consider the one-dimensional discrete time system:

$$
z_{i+1}=g\left(z_{i}\right):=\Xi_{q}\left(z_{i}\right) z_{i}
$$

we have that $\left\|c_{i}\left(\tau_{i}\right)\right\| \leq z_{i-1}$ when $z_{0}=\left\|c_{1}\left(\tau_{1}\right)\right\|$ by the properties of $\Xi_{q}$ given in Lemma 1. Moreover, by (8) and the fact that $W(0)=0$ (cf. [7]), the Jacobian of $g$ at 0 is given by:

$$
\left.\frac{\partial g(z)}{\partial z}\right|_{z=0}=\Xi_{q}(0)=-\frac{\left(f_{q}(0)\right)_{2}}{\left(f_{q}(0)\right)_{1}}<1 .
$$

Therefore, there exist $M \geq 1$ and $0<\gamma<1$ such that $z_{i} \leq M\left|z_{0}\right| \gamma^{i}$ for $\left|z_{0}\right|$ sufficiently small. It follows that when $z_{0}=\left\|c_{1}\left(\tau_{1}\right)\right\|$, for all $i \in \mathbb{N} \backslash\{0\}$,

$$
\left\|c_{i}\left(\tau_{i}\right)\right\| \leq z_{i-1} \leq M\left|z_{0}\right| \gamma^{i-1}=M\left\|c_{1}\left(\tau_{1}\right)\right\| \gamma^{i-1} .
$$

\section{REFERENCES}

[1] A. D. Ames, "A categorical theory of hybrid systems," Ph.D. dissertation, University of California, Berkeley, 2006.

[2] A. D. Ames, A. Abate, and S. Sastry, "Sufficient conditions for the existence of Zeno behavior," in 44th IEEE Conference on Decision and Control and European Control Conference, Seville, Spain, 2005.

[3] A. D. Ames and S. Sastry, "Characterization of Zeno behavior in hybrid systems using homological methods," in 24th American Control Conference, Portland, OR, 2005.

[4] A. D. Ames, P. Tabuada, and S. Sastry, "On the stability of Zeno equilibria," in Hybrid Systems: Computation and Control, ser. Lecture Notes in Computer Science, J. Hespanha and A. Tiwari, Eds., vol. 3927. Springer-Verlag, 2006, pp. 34-48.

[5] M. S. Branicky, "Stability of hybrid systems: State of the art," in 36th IEEE Conference on Decision and Control, San Diego, CA, 1997.

[6] E. A. Coddington and N. Levinson, Theory of Ordinary Differential Equations. McGraw-Hill, 1955.

[7] R. M. Corless, G. H. Gonnet, D. E. G. Hare, D. J. Jeffrey, and D. E. Knuth, "On the Lambert W function," Advances in Computational Mathematics, vol. 5, pp. 329-359, 1996.

[8] J. M. Esposito, V. Kumar, and G. J. Pappas, "Accurate event detection for simulating hybrid systems," in Hybrid Systems: Computation and Control, ser. Lecture Notes in Computer Science, M. D. D. Benedetto and A. L. Sangiovanni-Vincentelli, Eds., vol. 2034. Springer-Verlag, 2001, pp. 204-217.

[9] M. Heymann, F. Lin, G. Meyer, and S. Resmerita, "Analysis of Zeno behaviors in a class of hybrid systems," IEEE Transactions on Automatic Control, vol. 50, no. 3, pp. 376-383, 2005.

[10] K. H. Johansson, J. Lygeros, S. Sastry, and M. Egerstedt, "Simulation of Zeno hybrid automata," in 38th IEEE Conference on Decision and Control, Phoenix, AZ, 1999.

[11] H. K. Khalil, Nonlinear Systems, 2nd ed. Prentice Hall, 1996.

[12] J. Lygeros, K. H. Johansson, S. N. Simic, J. Zhang, and S. Sastry, "Dynamical properties of hybrid automata," IEEE Transactions on Automatic Control, vol. 48, no. 1, pp. 2-17, 2003.

[13] D. Nesic and D. Liberzon, "A small-gain approach to stability analysis of hybrid systems," in 44th IEEE Conference on Decision and Control and European Control Conference, Seville, Spain, 2005.

[14] J. Zhang, K. H. Johansson, J. Lygeros, and S. Sastry, "Zeno hybrid systems," Int. J. Robust and Nonlinear Control, vol. 11, no. 2, pp. 435-451, 2001. 